\title{
Does Vitamin D Protect the Gut Mucosal Barrier? Mechanistic Insights from Experimental Colitis
}

\author{
Maria O'Sullivan ${ }^{1}$
}

Received: 30 April 2015/Accepted: 6 May 2015/Published online: 19 May 2015

(C) Springer Science+Business Media New York 2015

There is growing interest in how vitamin $\mathrm{D}$ and the vitamin $\mathrm{D}$ receptor (VDR) regulate immune responses in inflammatory bowel disease (IBD), a topic addressed by Golan et al. [1] in this issue of Digestive Diseases and Sciences. Vitamin D deficiency, measured by low circulating serum 25-hydroxyvitamin D concentration, is common in IBD and may correlate with higher disease risk, severity, and relapse rates, suggesting that targeting vitamin D pathways may provide the basis for novel therapeutic strategies [2, 3]. This hypothesis is supported by data obtained from animal models, in which vitamin D deficiency accelerates the development of experimental colitis, whereas vitamin $\mathrm{D}$ treatment protects against colitis and attenuates inflammation [4, 5]. The VDR is expressed in multiple cell types including $\mathrm{T}$ cells, macrophages, and intestinal epithelial cells. There is a considerable body of evidence from murine models of colitis indicating that the immune modulatory effects of vitamin $\mathrm{D}$ on $\mathrm{T}$ cells are mediated through the VDR [6]. Vitamin D may also regulate intestinal barrier function [7-9], although the mechanism by which vitamin D alters the intestinal barrier in IBD is unclear. In line with this, Golan et al. [1] report their data on vitamin D/VDR-mediated mechanisms in maintaining gut barrier function in experimental colitis.

Increased intestinal permeability and impaired barrier function, well-documented features of human and murine IBD, have been implicated in disease pathogenesis and clinical relapse [10]. Building on past work [7, 8], Golan

Maria O'Sullivan

maria.osullivan@tcd.ie

1 Department of Clinical Medicine, Trinity College Dublin Centre for Health Sciences, St James's Hospital, Dublin 8, Ireland et al. [1] report that intestinal inflammation was attenuated through upregulation of epithelial VDR signaling. In the study, the authors introduced the human (h) VDR protein into intestinal epithelial cells obtained from the interleukin10 knockout (IL-10 KO) mouse model of colitis which expressed the hVDR transgene specifically in intestinal epithelial cells (IL-10 KO/Tg) and thus overexpressed VDR relative to IL-10 KOs. Of note, significantly reduced intestinal inflammation and reduced intestinal epithelial apoptosis were present in the IL-10 KO/Tg mice, supporting the hypothesis that increasing hVDR expression in intestinal epithelial cells attenuated colitis via epithelial VDR signaling. The findings broadly support recent work by the author group reporting that gut epithelial VDR signaling inhibited experimental colitis [8].

In interpreting the findings, the authors also propose that the overexpression of the hVDR strengthened the integrity of the epithelial barrier. Indeed, this study has nicely demonstrated that targeting epithelial VDR signaling mechanisms appears to reduce intestinal inflammation. Based on the present study alone [1], however, the assertion that this reduction in intestinal inflammation is a direct result of a VDR-mediated permeability reduction in the intestinal barrier is not supported based on the data presented. In this respect, direct evidence to support changes in barrier integrity such as changes in transepithelial electrical resistance (TER), measurement of permeability to small molecules, or expression of epithelial junctional proteins would be required. For example, determining expression and/or localization of members of the zonula occludens (ZO), claudin, or junctional adhesion molecule families would help corroborate the hypothesis that the VDR-mediated inhibition of colitis was achieved through strengthening the gut mucosal barrier. Although not investigated in the present study [1], similar work from the 
author group has reported that vitamin D through VDR signaling [8] maintained TER while increasing ZO-1 and claudin-1 expression.

The findings of Golan et al. [1] viewed in the context of others $[3,8]$ suggest that targeting intestinal epithelial VDR signaling may ameliorate inflammation in experimental colitis. Although this effect may in part be due to the alteration of intestinal barrier function, given the widespread expression of the VDR on numerous cells other than gut epithelial cells, such as immune cells (including chronically activated T cells [6]), effects are unlikely to be confined to the epithelial VDR alone.

The challenge is to determine how these data translate to human IBD, where hypothesis testing becomes more complex due to the many uncontrolled variables present. Our recent pilot intervention study [9] suggests that although oral vitamin D may maintain intestinal permeability in Crohn's disease, the effects were modest. In IBD, it is also argued that increased intestinal permeability and barrier dysfunction may not represent a primary defect but rather reflect the underlying disease [10]; equally, this "reverse causality" argument holds for low vitamin D status in IBD [3].

The findings presented here [1] are noteworthy and add support to the growing interest in targeting the VDR as a therapeutic strategy in IBD, either through vitamin $\mathrm{D}$ treatment [2, 9] or through anti-inflammatory therapy such as antitumor necrosis factor-directed therapies. In the future, investigations into the contributions of other proposed mechanisms such as autophagy [11] or antimicrobial peptides and the gut microbiome [3, 11] toward vitamin D/VDR signaling in colitis may offer further insights into the pathogenesis and treatment of IBD.

\section{References}

1. Golan MA, Liu W, Shi Y, et al. Transgenic expression of vitamin $\mathrm{D}$ receptor in Gut epithelial cells ameliorates spontaneous colitis caused by interleukin-10 deficiency. Dig Dis Sci. (Epub ahead of print). doi:10.1007/s10620-015-3634-8.

2. Jorgensen SP, Agnholt J, Glerup H, et al. Clinical trial: vitamin D3 treatment in Crohn's disease-a randomized double-blind placebo-controlled study. Aliment Pharmacol Ther. 2010;32: 377-383.

3. O'Sullivan M. Vitamin D as a novel therapy in inflammatory bowel disease: new hope or false dawn? Proc Nutr Soc. 2015;74: $5-12$.

4. Cantorna MT, Munsick C, Bemiss C, et al. 1,25-Dihydroxycholecalciferol prevents and ameliorates symptoms of experimental murine inflammatory bowel disease. J Nutr. 2000;130: $2648-2652$.

5. Zhu Y, Mahon B, Froicu M, et al. Calcium and 1,25-dihydroxyvitamin D3 target the TNF-a pathway to suppress experimental inflammatory bowel disease. Eur J Immunol. 2005;35:217-224.

6. Cantorna MT, Waddell A. The vitamin D receptor turns off chronically activated T cells. Ann N Y Acad Sci. 2014;1317: 70-75.

7. Kong J, Zhang Z, Musch MW, et al. Novel role of the vitamin D receptor in maintaining the integrity of the intestinal mucosal barrier. Am J Physiol Gastrointest Liver Physiol. 2008;294:G208$\mathrm{G} 216$.

8. Liu W, Chen Y, Golan MA, et al. Intestinal epithelial vitamin D receptor signaling inhibits experimental colitis. J Clin Investig. 2013;123:3983-3996.

9. Raftery T, Martineau AR, Greiller CL, et al. Effects of vitamin D supplementation on intestinal permeability, cathelicidin and disease markers in Crohn's disease: results from a randomised doubleblind placebo-controlled study. United European Gastroenterol J. 2015. doi:10.1177/2050640615572176.

10. Odenwald MA, Turner JR. Intestinal permeability defects: is it time to treat? Clin Gastroenterol Hepatol. 2013;11:1075-1083.

11. Wu S, Zhang YG, Lu R, et al. Intestinal epithelial vitamin D receptor deletion leads to defective autophagy in colitis. Gut. 2014. doi:10.1136/gutjnl-2014-307436.

Conflict of interest None. 